\title{
Breast Cancer Chemotherapy and Treatments
}

\author{
Li Pin $\mathrm{Kao}^{1,2 *}$ \\ ${ }^{1}$ Department of Basic Medical Sciences, Purdue University, Indiana, USA \\ ${ }^{2}$ Centre for Cancer Research, Purdue University, Indiana, USA \\ *Corresponding author: Li Pin Kao, Department of Basic Medical Sciences, Centre for Cancer Research, Purdue University, USA
}

Submission: 睯February 02, 2018; Published: 眥August 17, 2018

\begin{abstract}
Breast cancer, the first most common malignant tumor in women worldwide. Many etiological factors such as a wide spectrum of clinical manifestations caused by family clustering, hormonal factors, physiology changed, environmental inducers, and life styles. Although there are many FDA-approval drugs for breast cancers. Tumors are heterogenous and individual has inherited different genetic background. Thus, it is hard to find an effective treatment and it may recurrent after recovery from first treatment.
\end{abstract}

Keywords: Breast cancer; Autophagy; Autophagy related inhibitors; Chemotherapy

\section{Introduction}

The mortality rate exceeds 144,000 cases and most of the breast cancer (BC) cases are resistant to traditional chemotherapy and radiotherapy. Wide varieties of chemotherapeutic agents have been tried and are in use, including tamoxifen, docetaxel, anthracyclines (i.e. doxorubicin, and epirubicin), taxanes (i.e. paclitaxel, and docetaxel), 5-fluorouracil, cyclophosphamide, and carboplatin. No regimen has been proved to be curative. Often, the response rate and prolongation of survival are minimal (a few months or less), and there is a significant morbidity associated with poor treatment effects. Surgical resection is considered the first-line options for early tumors, although there is no agreement on which the best approach is. Interestingly, triple negative has been reported has higher chemo-resistance than other type of breast cancers with low autophagy activity [1,2]. Antiestrogen resistant cell lines exhibit increased basal autophagy when compared with their antiestrogen sensitive parental cells [3]. Study has shown to manipulate transient receptor potential channel 5 (TRPC5), a $\mathrm{Ca}_{2}+$ permeable cation channel, which helps in promotes autophagic activity [4]. Studies also demonstrate the breast cancer usually Prosurvival after autophagy activity increase by various therapeutics [5-7]. Moreover, Tamoxifen and Faslodex (ICI) both induce autophagy in ER+ breast cancer cells without [3-11]. There are small molecules which has been in FDA-approval drug lists and applied in clinical trial in different diseases.

Antiestrogen resistant cell lines exhibit increased basal autophagy. Study had demonstrated ccombination of Tamoxifen or Faslodex (ICI) with Hydroxychloroquine (HCQ) had different anti-estrogen responsiveness in vitro or in vivo which may affect by tumor microenvironment (i.e. chemokines, macrophage development/activity [12]. Inhibiting autophagy via autophagyrelated genes (i.e. autophagy-related gene (Atg) 5, Atg7, and p62/ SQSTM1) silencing potentiated antiestrogen-mediated cell death, indicating that antiestrogen stimulated autophagy is prosurvival and a critical mechanism of therapy resistance. Overall, it indicated that increased in autophagy activity in early recurring breast cancer when compared with breast cancer that never recurs. Moreover, elevated p62 is significantly correlated with poor survival in breast cancer patients, suggesting a role for autophagy in breast cancer re occurrence. Manipulation of autophagy activity can be a potential therapy for chemotherapy in vitro or in vivo. Therefore, diagnosis or detection at an early stage is crucial to allow the application of treatments for increasing the life expectancy of the patient [13] (Table 1,2).

Table 1: Autophagy inhibitors during autophagy pathway.

\begin{tabular}{|c|c|c|}
\hline Name & Mechanism & phosphoinositide3-kinase (PI3) inhibitor \\
\hline 3-Methyladenine & PI3-kinase inhibitor & Auto phagosome formation \\
\hline Wort mannin & PI3-kinase inhibitor & Autophagosome formation \\
\hline LY294002 & Unc-51-like kinase 1 (ULK1) Inhibitor & Autophagosome formation \\
\hline SBI-0206965 & Nome formation & \\
\hline
\end{tabular}




\begin{tabular}{|c|c|c|}
\hline Spautin-1 & ubiquitin-specific peptidases (USP10) and (USP13) inhibitor & Autophagosome formation \\
\hline SAR405 & Vacuolar Protein Sorting Protein 18 and 34 (Vps18 and Vps34) inhibitor & Autophagosome formation \\
\hline NSC185058 & autophagy-related gene 4 (ATG4) inhibitor & Autophagosome formation \\
\hline Verteporfin & Unknown & $\begin{array}{c}\text { Autophagosome formation and accumu- } \\
\text { lation }\end{array}$ \\
\hline ROC325 & Unknown & Lysosome \\
\hline Lys05 & Unknown & Lysosome \\
\hline Chloroquine & Unknown & Lysosome \\
\hline Hydroxychloroquine & Unknown & Lysosome \\
\hline
\end{tabular}

Table 2: Current Hydroxychloroquine (HCQ) clinical trials.

\begin{tabular}{|c|c|c|c|}
\hline Treatment & Condition & Phase Trial & Reference \# at ClinicalTrials.gov \\
\hline HCQ + sunitinib malate & Adult solid neoplasm & I & NCT00813423 \\
\hline $\mathrm{HCQ}+$ vorinostat & Malignant solid tumour & I & NCT01023737 \\
\hline HCQ + sirolimus or vorinostat & Advanced cancers & I & NCT01266057 \\
\hline $\begin{array}{l}\text { HCQ + Protein kinase B (Akt) inhibitor } \\
\text { MK-2206 dihydrochloride (MK2206) }\end{array}$ & Advanced cancers & I & NCT01480154 \\
\hline HCQ as a single agent & Estrogen receptor positive breast cancer & I & NCT02414776 \\
\hline HCQ + gemcitabine & Advanced adenocarcinoma & $\mathrm{I} / \mathrm{II}$ & NCT01506973 \\
\hline HCQ + Interleukin 2(IL-2) & Renal cell carcinoma & $\mathrm{I} / \mathrm{II}$ & NCT01550367 \\
\hline $\mathrm{HCQ}+$ vorinostat & Colorectal cancer & $\mathrm{I} / \mathrm{II}$ & NCT02316340 \\
\hline HCQ + gemcitabine/carboplatin & Small cell lung cancer & $\mathrm{I} / \mathrm{II}$ & NCT02722369 \\
\hline HCQ + capecitabine & Pancreatic carcinoma & II & NCT01494155 \\
\hline HCQ as a single agent & Prostate cancer & II & NCT00726596 \\
\hline HCQ + Abraxane and gemcitabine & Pancreatic carcinoma & II & NCT01978184 \\
\hline
\end{tabular}

\section{Conclusion}

It is very little is known how the manipulation of a biological process toward to potential cancer therapy. As is often the case, we need understand the basic mechanistic of biological processes if this is going to apply for a clinical benefit or other practical application. This article demonstrated the importance of manipulation of autophagy activities in different cancer treatment in basic biomedical research and different clinical trials. Given the rapid progress in understanding autophagy in cancer-related researches and what it does, manipulation of autophagy activities in cancer-related research has ready to take off.

\section{Acknowledgement}

The author would like to acknowledge the financial support of the Purdue University for provided support in the form of salaries of KLP listed but did not have any additional role in the study design, data collection and analysis, decision to publish, or preparation of the manuscript.

\section{References}

1. Garbar C (2017) Chemotherapy treatment induces an increase of autophagy in the luminal breast cancer cell MCF7 but not in the triplenegative MDA MB231. Sci Rep 7(1): 7201.

2. O'Reilly EA (2015) The fate of chemo resistance in triple negative breast cancer (TNBC). BBA Clin 3: 257-275.
3. Cook KL (2012) Glucose regulated protein 78 controls cross talk between apoptosis and autophagy to determine antiestrogen responsiveness. Cancer Res 72(13): 3337-3349.

4. Zhang P (2017) TRPC5 induced autophagy promotes drug resistance in breast carcinoma via CaMKKbeta/AMP alpha/mTOR pathway. Sci Rep $7(1): 3158$.

5. Clarke R (2012) Endoplasmic reticulum stress the unfolded protein response autophagy and the integrated regulation of breast cancer cell fate. Cancer Res 72(6): 1321-1331.

6. Thomas S (2011) Addition of a histone deacetylase inhibitor redirects tamoxifen treated breast cancer cells into apoptosis which is opposed by the induction of autophagy. Breast Cancer Res Treat 130(2): 437-447.

7. Vazquez Martin A, C Oliveras Ferraros, JA Menendez (2009) Autophagy facilitates the development of breast cancer resistance to the anti HER2 monoclonal antibody trastuzumab. PloS One 4(7): e6251.

8. Cook KL (2014) Mitochondria directly donate their membrane to form autophagosomes during a novel mechanism of parkin associated mitophagy. Cell Biosci 4(1): 16.

9. Clarke R (2009) Gene network signalling in hormone responsiveness modifies apoptosis and autophagy in breast cancer cells. J Steroid Biochem Mol Biol 114(1-2): 8-20.

10. Schoenlein PV (2009) Autophagy facilitates the progression of ER alpha positive breast cancer cells to antiestrogen resistance. Autophagy 5(3): 400-403.

11. Samaddar JS (2008) A role for macroautophagy in protection against 4-hydroxytamoxifen-induced cell death and the development of antiestrogen resistance. Mol Cancer Ther 7(9): 2977-2987. 
12. Cook KL (2014) Hydroxychloroquine inhibits autophagy to potentiate antiestrogen responsiveness in ER+ breast cancer. Clin Cancer Res 20(12): 3222-3232.
13. Chude CI, RK Amaravadi (2017) Targeting Autophagy in Cancer: Update on Clinical Trials and Novel Inhibitors. Int J Mol Sci 18(6).
Creative Commons Attribution 4.0 International License

For possible submissions Click Here

MADD $\begin{gathered}\text { Modern } \\ \text { Aoproaches in } \\ \text { Drug Designing }\end{gathered}$
Benefits of Publishing with us
- High-level peer review and editorial services
- Freely accessible online immediately upon publication
- Authors retain the copyright to their work
- Licensing it under a Creative Commons license
- Visibility through different online platforms

